\title{
A General Method for Quantifying the Amorphous Phase in Nano Polycrystalline Materials
}

\author{
Mehran Masoudi ${ }^{12}$, Mansor Hashim ${ }^{2}$, Halimah Mohamed Kamari ${ }^{1} \&$ Mohd Sapuan Salit ${ }^{3}$ \\ ${ }^{1}$ Department of Physics, Faculty of Science, Universiti Putra Malaysia, Selangor, Malaysia \\ ${ }^{2}$ Institute of Advanced Technology, Universiti Putra Malaysia, Selangor, Malaysia \\ ${ }^{3}$ Department of Mechanical and Manufacturing Engineering, Universiti Putra Malaysia, Selangor, Malaysia \\ Correspondence: Mehran Masoudi, Department of Physics, Faculty of Science, Universiti Putra Malaysia; \\ Advanced Materials and Nanotechnology Laboratory, Institute of Advanced Technology, Universiti Putra \\ Malaysia, Serdang 43400 UPM, Selangor, Malaysia. Tel: 60-1-7848-7494. E-mail: mehranmasoudi@yahoo.com
}

The research is financed by (Advanced Materials and Nanotechnology Laboratory, Institute of Advanced Technology, Universiti Putra Malaysia, 43400 UPM Serdang, Selangor, Malaysia)

\author{
Received: April 5, $2012 \quad$ Accepted: April 25, $2012 \quad$ Online Published: May 10, 2012 \\ doi:10.5539/mas.v6n6p1 URL: http://dx.doi.org/10.5539/mas.v6n6p1
}

\begin{abstract}
The grain boundary area has a great influence on the polycrystalline material properties and in nano crystallized metals. It could be considered as a glass metal phase in addition to crystallites in a composite structure. In this research, a general procedure for directly quantifying the crystalline and amorphous phases is introduced by using an x-ray diffractometry technique.

Nickel with a nano crystalline structure was deposited onto pure copper samples with grain sizes of between 17 to $124 \mathrm{~nm}$ by an electroplating technique. A combination of measuring the area of the XRD pattern characteristic peaks and x-ray background scattering were used for the estimation of the crystalline and amorphous volume percentages. The results show that there is a non-linear relationship between the percentage of the crystalline phase calculated by the geometrical method and the area of the selected XRD peak. An experimental formula is suggested for the calculation of the crystalline phase volume percentage of the nano structure nickel samples based on the (200) x-ray peak area.
\end{abstract}

Keywords: nanostructures, amorphous materials, X-ray diffraction, electrochemical techniques, crystal structure

\section{Introduction}

For more than three decades, scientists have increasingly focused on designing new nano structured materials with novel behaviours and properties which sometimes are completely different from those of conventional coarse grain materials. The grain boundaries density of a nano material grows quickly along with decreasing grain sizeswhich improves the mechanical properties as it has been has been explained by the Hall-Petch formula for the materials with sub-micron grain sizes (Nieh \& Wang, 2005; Giga A. et al., 2006; Trelewicz \& Schuh, 2007).

The amorphous metal or a glass metal is a metallic material with a disordered atomic-scale structure. The glass metals are tough and have better resistance to wear and corrosion, lower thermal conductivity andmagnetic coercivity and higher electrical resistance compared to crystalline metals. The volume percentage of amorphous phase in grain boundaries is not negligible in nano scale crystalline materials and it means that in fact nano grain polycrystalline materials are a composite of crystal and amorphous phases. This could be one of the explanations for the special behaviours of ultra-fine grain materials (Bonetti \& Pasquini 1999; Grigorieva T. F. et al., 2003; Roland T. et al., 2006; Langdon T. G., 2010; Vincze I. et al., 1980; Löhneysen H. v., 1991).

Considering the importance of the grain boundaries volume in relation to the chemical, mechanical and physical properties of polycrystalline materials, an efficient and accurate quantification of the amorphous phase in a fine polycrystalline sample is a challenging task. X-ray diffraction has been already used for the quantification of mixtures with the help of internal standards for materials such as drugs, cements or ceramics (Montejo-Bernardo 
J. et al., 2005; Varlashkin P., 2011; Martello E. D. et al., 2011). In some techniques, the integrated intensities of some different peaks have been used for the quantification analysis of mixtures. This paper describes the relationship between the grain boundaries volume as an amorphous phase in a nano structured metal with its $\mathrm{x}$-ray diffraction pattern without using internal standards.

\section{Experimental Aspects}

Nickel films were electroplated onto pure copper samples by applying a constant current in a typical Watts plating solution. Seven nickel coated samples were prepared under different plating conditions. The samples were respectively from minor to major grain sizes labelled N1 to N7. As already reported by other researchers, by increasing the current density and by adding neutral fine powers to the electrolyte, the rate of nickel nucleation can be increased (Thiemig \& Bund, 2008; Gül K. et al., 2009). Based on this fact, the samples were prepared in a nickel electroplating cell with chemical composition and electroplating parameters as shown in Table 1.

Table 1. Electroplating bath composition and parameters

\begin{tabular}{cc}
\hline Component & Concentration $(\mathrm{g} / \mathrm{l})$ \\
\hline $\mathrm{NiSO}_{4} \cdot 6 \mathrm{H}_{2} \mathrm{O}$ & 240 \\
$\mathrm{NiCl}_{2} \cdot 6 \mathrm{H}_{2} \mathrm{O}$ & 40 \\
$\mathrm{H}_{3} \mathrm{BO}_{3}$ & 350 \\
$\mathrm{CH}_{3}\left(\mathrm{CH}_{2}\right)_{11} \mathrm{OSO}_{3} \mathrm{Na}$ & 0.2 \\
Deposition Condition & Value \\
\hline $\mathrm{pH}$ & 4 \\
Time $(\min )$ & 60 \\
Temperature $\left({ }^{\circ} \mathrm{C}\right)$ & 45 \\
Current density $\left(\mathrm{A} / \mathrm{dm}^{2}\right)$ & 3 to 10 \\
\hline
\end{tabular}

The $\mathrm{pH}$ of the electrolyte was regulated between 4.0 to 4.2 by adding $0.1 \mathrm{~mol} \mathrm{HCl}$ and $0.05 \mathrm{~mol} \mathrm{NaOH}$ solutions based on $\mathrm{pH}$ fluctuations. The electrolyte was mechanically stirred constantly at $350 \mathrm{rpm}$ using a magnetic bar. Samples with dimensions of $25 \times 10 \times 1 \mathrm{~mm}$ were cut off from a commercial pure copper plate and all samples were polished with silicon carbide papers up to grade 2000 grit, cleaned in a $0.1 \mathrm{~mol} \mathrm{NaOH}$ solution and activated in 5\% Hydrochloric acid at room temperature. The anode was a pure nickel (99.98\%) plate with an area of $10 \mathrm{~cm}^{2}$. The temperature of the electrolyte was maintained at $45^{\circ} \mathrm{C}$ by an automatic temperature controller (PLT, Scientific Company - accuracy $\pm 1^{\circ} \mathrm{C}$ ). The plating was conducted at a current density of 3 to $10 \mathrm{~A} / \mathrm{dm}^{2}$ for 60 minutes using a DC power supply (EKK-AJ5D) with \pm 0.05 A current accuracy.

The microstructure of the electroplated coated surfaces and cross sections were studied by field emission high resolution scanning electron microscopy (FEI, Nova Nano SEM - 2300). The crystalline structure and grain size of the nickel layers were determined by X-ray diffractometry $(\mathrm{Cu}-\mathrm{K} \alpha)$ at roomtemperature and by applying the Scherrer formula. The X-ray scan rate was $0.15 \mathrm{~min}^{-1}$ over a $2 \theta$ range of 10 to 90 degrees.

\section{Results \& Discussion}

\subsection{Nicklenanocrystalline Samples}

The SEM micrographs in Figure 1 show the surface and cross section of the nickel layer (sample N3) on a copper base. The mean thickness of the nickel layer was determined to be 34 microns. The nickel grain sizes were estimated using SEM observation and micrograph image analysis to support the grain size figure calculated using an X-ray diffractometry technique. The microscopic studies confirmed the formation of a fine nickel grain structure on the surface of the coated samples. However the grain sizes calculated by using the XRD pattern technique were considered as the main reference data for further studies. 


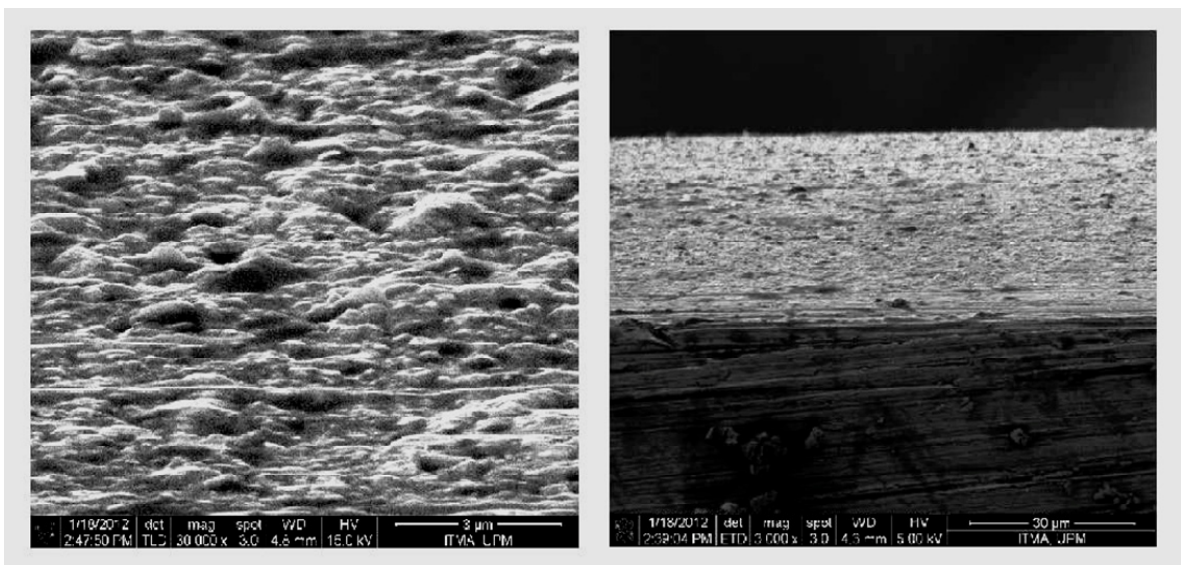

Figure 1. Nickel electroplated layer: (a) top surface 30,000X and (b) cross section 3000X, SEM micrographs

The XRD patterns of all samples were matched with the face-centred cubic (FCC) reference peaks of nickel (Figure 2).

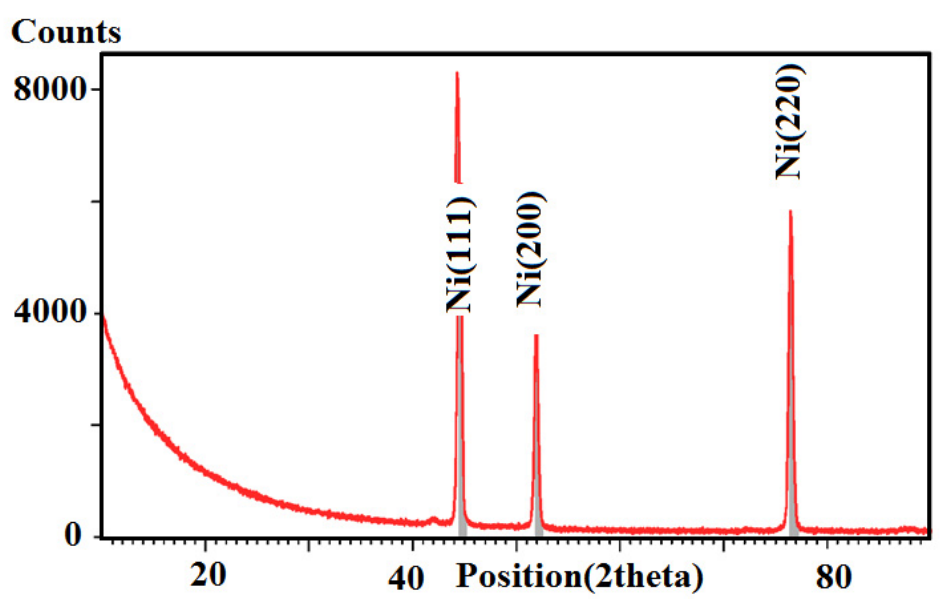

Figure 2. XRD pattern of nickel coating (Sample N2)

The average particle size of the coating layers were calculated by using the X-Ray diffraction characterization (200) peak located at $2 \theta \approx 51.83$ in the Scherrer equation, $D=K \lambda /(\beta \cdot \cos \theta)$, disregarding the effect of the microstrains: $K$ is the shape factor (considered as 0.9 ), $\lambda$ is the x-raywavelength, typically $1.54 \AA, \beta$ is the line broadening at half the maximum intensity (FWHM) of the selected XRD reflection peak in radians, and $\theta$ is the Bragg angle; D is the mean size of the crystalline domains which may be smaller or equal to the real grain size. The grain sizes were calculated to be between 17 and $90 \mathrm{~nm}$ as shown in Table 2 .

Table 2. Calculated Grain size and strain of samples by using Scherrer and Hall-Pitch formulas

\begin{tabular}{cccc}
\hline Sample & $\begin{array}{c}\text { GrainSize }(\mathrm{nm}) \\
\text { Scherrer formula }\end{array}$ & $\begin{array}{c}\varepsilon \text {.Starin } \\
\text { H-P formula }\end{array}$ & Grain size $(\mathrm{nm})$ \\
\hline N1 & 17 & 0.016 & 17 \\
N2 & 18 & 0.011 & 18 \\
N3 & 21 & 0.034 & 23 \\
N4 & 25 & 0.0134 & 26 \\
N5 & 25 & 0.025 & 27 \\
N6 & 41 & 0.033 & 48 \\
N7 & 90 & 0.027 & 124 \\
\hline
\end{tabular}


The microstrain $(\mathcal{E})$ of the nickel layers was calculated by using following relationship:

$$
\boldsymbol{\beta}_{\text {observation }}^{2}=\boldsymbol{\beta}^{2}{ }_{\text {Actual }}+\boldsymbol{\beta}_{\text {Instrumental }}^{2}+\boldsymbol{\beta}^{2}{ }_{\text {strain }}
$$

where peak broadening caused by microstrains, $\beta_{\text {strain }}$ is equal to $4 . \mathcal{E} \cdot \tan (\theta)$ and $\beta_{\text {Instrumental }}$ is considered constant (estimated to be about 0.05 degrees). The calculated $\beta_{\text {Actual }}$ was used to recalculate the sample grain sizes (Table 2) and the results show that the error in the calculation of the grain sizes increases with growing grain size.

The grain boundary volume percentage for grain sizes between 1 to $150 \mathrm{~nm}$ was calculated by postulating that the grain boundary width was $2 \mathrm{~nm}$. The grain shape was considered as a truncated octahedron and the amorphous phase volume percentage was calculated for grain sizes smaller than $100 \mathrm{~nm}$ as shown in graph of Figure 3. The result indicates that for a sample with a $20 \mathrm{~nm}$ grain size, the glass metal phase volume percentage is more than $42 \%$ which is a considerable volume besides the crystalline phase.

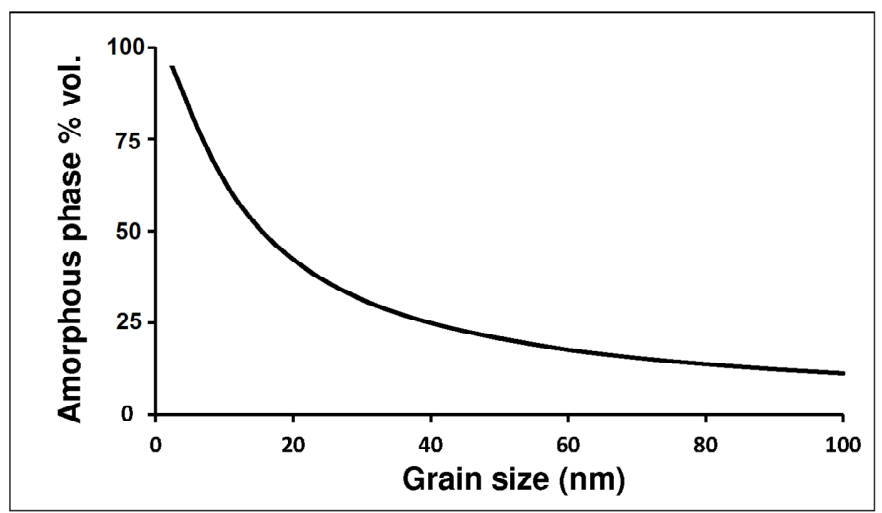

Figure 3. Calculated amorphous volume percentage versus grain sizes

\subsection{Quantitative Analysis}

The analysis of the X-RD profiles obtained in this study was based on the selected peak area devoid of the background area which was considered to be amorphous phase X-ray scattering. Two sets of base line data about 2 degrees on either side of the selected peak (200) were used to calculate the background average intensity. This was to minimize any angular dependence in the background figure and moreover, the limits were considered to be near to the selected peak and not belonging to the crystalline peak profile. The area of main peak was calculated by using the following formula:

$$
\text { Peak Area }=0.5 * \text { FWHM * (Peak intensity - background intensity })
$$

The graph of Figure 4 shows the (200) peak area versus the grain sizes. The curve portrays a non-linear relationship between these two parameters where increasing the grain size reduces the peak area. Using the calculated crystalline volume percentage (Figure 3), the (200) peak area plotted versus the crystalline phase percentage is shown in Figure 5, and this curve also indicates a good non-linear relationship between the two quantities. Using the same method, the percentage of the amorphous phase (grain boundary volume) is graphed against the (200) peak area in Figure 6 and the same strong correlation is observed. In data analysis and calculations, the value for sample N4 was rejected as being very different from the rest. The experimental results were used to fit the following exponential curve to the volume percentage of the crystalline phase versus the peak area.

$$
P a=E X P\left(A-B . \operatorname{LnC} C_{v}\right)
$$

Where $P a$ is the peak area minus the background, $C_{\mathrm{v}}$ is the crystalline phase percentage, $A$ and $B$ are constants calculated to be 26.9 and 5.78 respectively. This relationship gives an estimation of the crystalline (or amorphous) phase of a nickel nano polycrystalline structure in a range of about 15 to $150 \mathrm{~nm}$ with a 4.9 RMSE (root mean square error). 


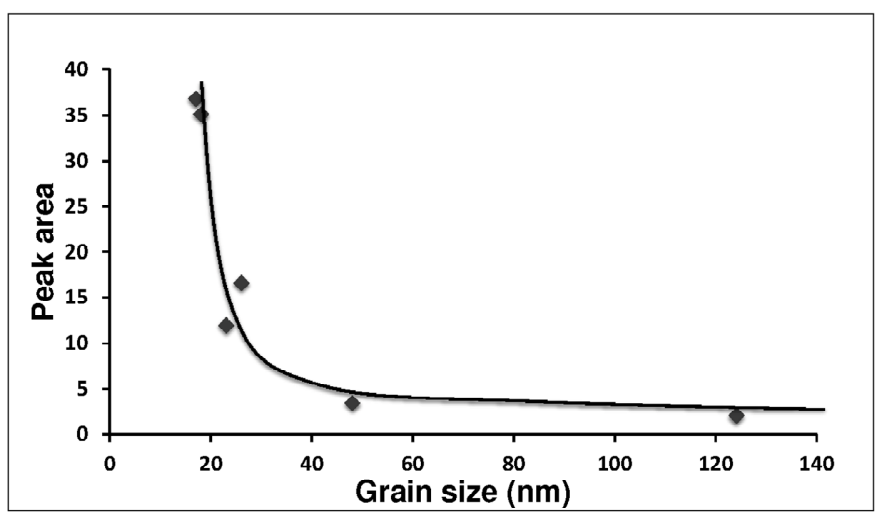

Figure 4. Nickel (200) peak area versus grain sizes

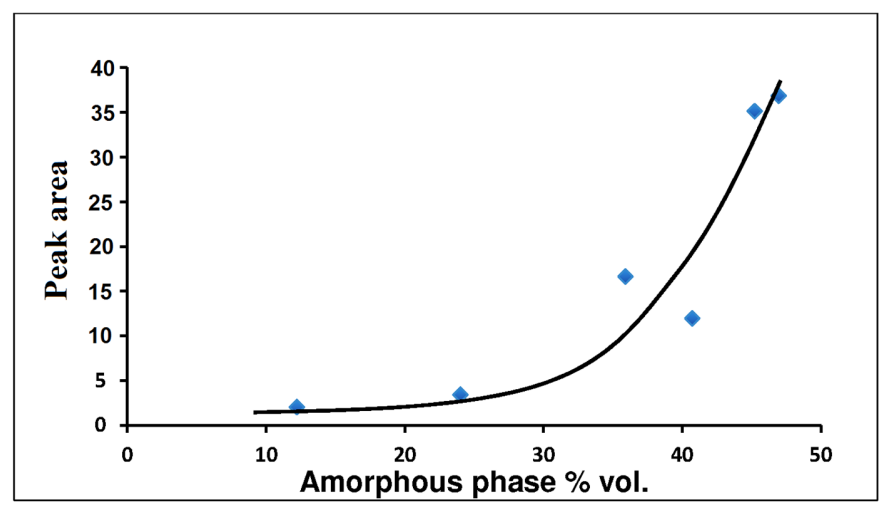

Figure 5. Nickel (200) peak area versus amorphous phase \% vol

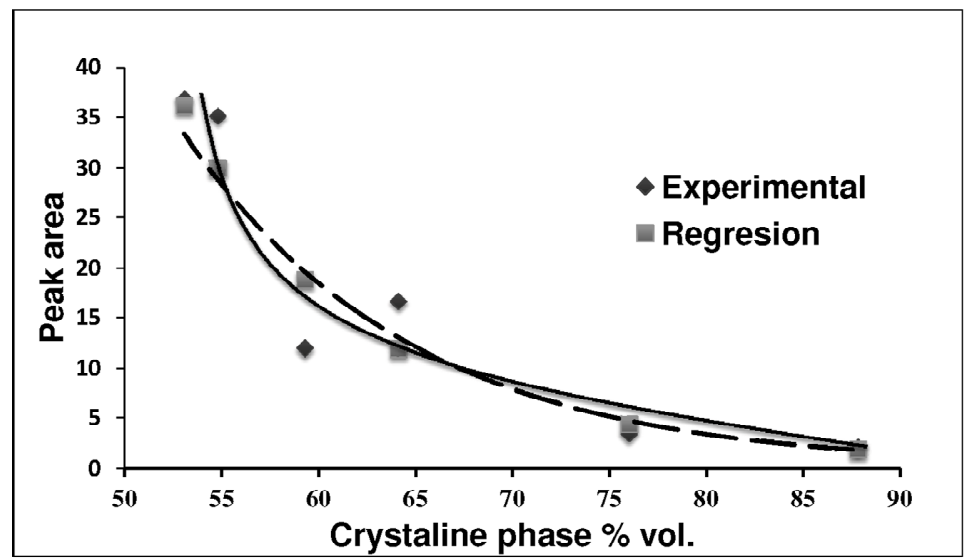

Figure 6. Nickel (200) peak area versus crystalline phase volume curves

\subsection{Advantages and Disadvantages}

The presented technique for quantifying the grain boundaries density of polycrystalline samples is a novel technique in this area which is based on applying experimental results of XRD spectroscopy tests without internal standard. By using this technique the relationship between the characteristic X-ray peak area and the volume percentage of amorphous phase related to grain boundaries can be formulated. However, this method needs to standard samples for each material prior to formulate the amorphous phase volume percentage based on selected characteristic x-ray diffraction peak. In next step, it is possible to study nano crystalline material properties based on composite materials behaviour and the result of explained technique in this study. The limits of detection and quantification of the presented method are in the range of about 15 to $100 \mathrm{~nm}$ only, as the samples tested with lower grain sizes have not yet been studied and applying the Scherrer formula to calculate 
the grain size above $100 \mathrm{~nm}$ is not recommended.

\section{Conclusion}

An easy quantification method has been suggested to quantify the volume percentage of the crystalline and amorphous phases in nano structured polycrystalline materials. This is based on the X-ray diffraction area of the selected peak and an estimation of the background scattering intensity as two measurable quantities. The studies on the nickel electroplated layers with different grain sizes show a non-linear relationship between the two quantities which could be used to define a simple experimental formula based on using regression methods. The procedure can easily be applied to other polycrystalline samples to estimate the amorphous phase volume percentage and could be important to further related studies on polycrystalline material properties.

\section{Acknowledgements}

This research was supported by the Advanced Materials and Nanotechnology Laboratory, Institute of Advanced Technology (ITMA) and the Department of Physics, Faculty of Science, Universiti Putra Malaysia (UPM).

\section{References}

Bonetti, E., \& Pasquini, L. (1999). Mechanical spectroscopy of nanocrystalline metals: Structure and anelasticbehaviour. J. Electronic Materials, 28(9), 1055-1061. http://dx.doi.org/10.1007/s11664-999-0183-y

Giga, A., Kimoto, Y., Takigawa, Y., \& Higashi, K. (2006). Demonstration of an inverse Hall-Petch relationship in electrodeposited nanocrystalline Ni-W alloys through tensile testing. ScriptaMaterialia, 55(2), 143-146. http://dx.doi.org/10.1016/j.scriptamat.2006.03.047

Grigorieva, T. F., Barinova, A. P., \& Lyakhov, N. Z. (2003). Mechanosynthesis of Nanocomposites. J. Nanoparticle Research, 5(5), 439-453. http://dx.doi.org/10.1023/B:NANO.0000006093.26430.3b

Gul, H., \& Killc, F. (2009). Characteristics of electro-co-deposited Ni- $\mathrm{Al}_{2} \mathrm{O}_{3}$ nano-particle reinforced metal matrix composite (MMC) coatings. Wear, 267(5-8), 976-990. http://dx.doi.org/10.1016/j.wear.2008.12.022

Langdon, T. G. (2010). The impact of bulk nanostructured materials in modern research. Adv. Mater. Sci., 25 , $1-11$.

Löhneysen, H. v. (1991). Thermal properties of amorphous metals. Materials Science and Engineering, A., 133, 51-58. http://dx.doi.org/10.1016/0921-5093(91)90012-C

Martello, E. D., Gabriella T., Raaness, O., \& Arnberg, L. (2011). Combined XRD and XRF Technique for the Quantification of the mass balance in a Si Carbothemic production Experiment. ISIJ Int., 51(9), 1492-1496. http://dx.doi.org/10.2355/isijinternational.51.1492

Montejo-Bernardo, J., García-Granda, S., Bayod-Jasanada, M., Llorente, I., \& Llavona, Luján. (2005). An easy and general method for quantifing Azithromycin dihydrate in a matrix of amorphous Azithromycin. Santiago Garcia-Granda, Arkivoc.ix, 321-331.

Nieh, T. G., \& Wang, J. G. (2007). Hall-Petch relationship in nanocrystalline Ni and Be-B alloys. Intermetallics, 13(3-4), 377-385. http://dx.doi.org/10.1016/j.intermet.2004.07.029

Roland, T., \& Retraint, D. (2006). Fatigue life improvement through surface nanostructuring of stainless steel by means of surface mechanical attrition treatment. ScriptaMaterialia, 54(11), 1949-1954. http://dx.doi.org/10.1016/j.scriptamat.2006.01.049

Roland, T., \& Retraint, D. (2007). Enhanced mechanical behavior of a nanocrystallised stainless steel and its thermal stability. Materials Science and Engineering, A., 445(446), 281-288. http://dx.doi.org/10.1016/j.msea.2006.09.041

Thiemig, D., \& Bund, A. (2008). Characterization of electrodeposited Ni- $\mathrm{TiO}_{2}$ nanocomposite coatings. Surface and Coatings Technology, 202(13), 2976-2984. http://dx.doi.org/10.1016/j.surfcoat.2007.10.035

Trelewicz, J. R., \& Schuh, C. A. (2007). The Hall-Petch breakdown in nanocrystalline metals: A crossover to glass-like deformation. ActaMaterialia, 55(17), 5948-5958. http://dx.doi.org/10.1016/j.actamat.2007.07.020

Varlashkin, P. (2011). Approaches to Quantification of Amorphous Content in Crystalline Drug Substance by Powder X-ray Diffraction. American Pharmaceutical Review, 14(1).

Vincze, I., \& Van Der Woude, F. (1980). Magnetic properties of amorphous transition metal alloys. J. Magnetism and Magnetic Materials, 15(18), 1336-1338. http://dx.doi.org/10.1016/0304-8853(80)90311-X 